\title{
Impact of Initial Electron Development on Sodium Plasma Generation Using LIBORS Technique: Numerical Modeling
}

\author{
Kholoud A. Hamam ${ }^{1}$, Yosr E. E.-D. Gamal ${ }^{2}$ \\ ${ }^{1}$ Department of Physics, Faculty of Science, King Abdulaziz University, Jeddah, KSA \\ ${ }^{2}$ National Institute of Laser Enhanced Sciences, Cairo University, El Giza, Egypt \\ Email: *khamam@kau.edu.sa
}

How to cite this paper: Hamam, K.A. and Gamal, Y.E.E.-D. (2018) Impact of Initial Electron Development on Sodium Plasma Generation Using LIBORS Technique: Numerical Modeling. Journal of Modern Physics, 9, 669-684.

https://doi.org/10.4236/jmp.2018.94046

Received: February 12, 2018

Accepted: March 27, 2018

Published: March 30, 2018

Copyright $\odot 2018$ by authors and Scientific Research Publishing Inc. This work is licensed under the Creative Commons Attribution International License (CC BY 4.0).

http://creativecommons.org/licenses/by/4.0/

\begin{abstract}
The present work reports an investigation on the role played by $\mathrm{Na}^{3+}$ ions formed through triatomic associative ionization collision of $\mathrm{Na}(4 \mathrm{~d})$ atoms with $\mathrm{Na}_{2}$ ground state molecules during the early phase of sodium plasma generation by laser ionization based on resonance saturation (LIBORS). Such ionization mechanism is observed experimentally for the first time by Tapalian and Smith (1993) [1]. In their experiment, stepwise atomic excitations are created using two CW dye lasers; one laser is tuned to $589 \mathrm{~nm}$ to excite the $\mathrm{Na}(3 \mathrm{~s})$ to $\mathrm{Na}(3 \mathrm{p}) \mathrm{D} 2$ transition of sodium and the other laser is tuned $569 \mathrm{~nm}$ to excite the $\mathrm{Na}(3 \mathrm{p})$ to $\mathrm{Na}(4 \mathrm{~d})$ transition. The analysis is grounded on a numerical study of the role of seed electron processes on the temporal evolution of sodium plasma formation by laser irradiation. A previously developed numerical model based on LIBORS technique is modified and adopted. In the present study, the sodium atom is treated as an atom comprises 22 levels namely: a ground state, 18 excited states and three ionic states (atomic, molecular and tri-atomic). The model tackled various collisional and radiative processes that act to enhance and deplete the free electrons generated in the interaction region. The contribution of these processes is signified by solving numerically a system of time-dependent rate equations, which couple the generated atomic and ionic species with the laser fields. Meanwhile, it solves the time-dependent Boltzmann equation for the electron energy distribution function (EEDF) of the generated electrons. The computed values of the EEDF, time evolution of both excited states population and the formed ionic species considering the individual effect of associative ionization, Penning, and photo-ionization and triatomic associative ionization justified the important effect of each of these ionizing processes in creating the early stage electrons. These seed electrons are assumed to rapidly gain energy through superelastic collisions leading eventually to plasma development.
\end{abstract}




\section{Keywords}

Plasma, Laser, Collisional Ionization, Association Ionization,

Tri-Atomic Ions, Photoionization, Electron Energy Distribution Function

\section{Introduction}

Study of laser-produced plasmas is one of the rapidly growing fields of present-day physics. The plasma generated by intense laser pulses in gases is a very well recognized phenomenon. The productivity of this process was found to be increased by many orders of magnitude when the laser wavelength is tuned to a selected transition of alkali vapors leading to fast ionization and plasma generation. Such phenomenon involves many excitation and ionization collisions besides radiative transfer processes.

The pioneering observation of this phenomenon was given by McIlrath and Lucatorto [2] when measurements are performed with laser radiation tuned to the first resonance line in dense sodium vapor. These measurements included some experimental study of the collisional mechanisms responsible for complete ionization in a sodium vapor illuminated by a CW laser radiation of wavelength $\lambda=589.0 \mathrm{~nm}$ tuned to the $3 \mathrm{~s}-3 \mathrm{p}$ resonance line. Ever since efficient ionization by resonance radiation has been tested in different alkali-metal vapors [3] [4] [5] [6]. The innovative work given in Ref. 2, has been extended by various experimental [7]-[12], and theoretical [13]-[19] studies. All aimed to investigate the excitation energy transfer and collisional ionization processes in a laser-excited alkali vapor. The investigation offered good evidence for their importance in radiative transfers involved different subjects such as: in gas lasers, astrophysics, controlled thermonuclear plasma, plasma physics, photochemistry [20] [21] as well as in creating plasma guide channels [22].

The results of McIlrath and Lucatorto [2] were interpreted by Measures et al., [22] [23], on the basis of superelastic heating of electrons in colliding with the excited atoms (collisions of the second kind). The theoretical model that describes the formation of resonance plasma is referred to as LIBORS. It proceeds as follows: initially, the $\mathrm{CW}$ laser field saturates the atomic resonance transition. Then free electrons are produced in the medium due to associative and Penning ionization processes [12]. These electrons gain energy in superelastic collisions by deactivating excited atoms, with the subsequent production of an electron avalanche resulting in complete ionization of the gas.

On the other hand, ionization of alkali atoms by nanosecond resonant laser pulses has played a strong part in coupling energy into vapor. Thus the feedback of resonant irradiation of dense sodium vapor, by nanosecond pulsed laser, has been the subject of some investigations by different researchers [24] [25] [26]. These studies revealed that enormous atomic, molecular, and photon interactions could take place in the exciting medium. Of specific importance are the io- 
nization mechanisms and energy transfer processes which are acting to enhance the electrons density leading eventually to plasma formation. Theoretical modeling of ionization of sodium vapor by nanosecond resonant laser pulses tuned to the 3s-4p transition, is presented by Stacewicz and Latek [27]. In this study, the laser pulse saturates this selected optical transition and produces a small number of initial electrons. Then the avalanche ionization develops without the presence of the laser radiation, using the energy stored in the excited atoms.

On the other hand, in (1993) Tapalian and Smith [1] observed experimentally for the first time the formation of $\mathrm{Na}^{3+}$ ions. These ions are produced through triatomic associative ionization interaction between $\mathrm{Na}(4 \mathrm{~d})$ atoms and $\mathrm{Na}_{2}$ ground state molecules (which are assumed to be present at $\approx 0.5 \%$ percent in the atomic beam), during sodium plasma generation by laser ionization based on resonance saturation (LIBORS), according to the reaction

$$
\mathrm{Na}(4 \mathrm{~d})+\mathrm{Na}_{2} \rightarrow \mathrm{Na}_{3}^{+}+\mathrm{e}^{-}
$$

The manifestation of this process requires a high population of the $\mathrm{Na}(4 \mathrm{~d})$ state. Therefore, in these measurements, stepwise atomic excitations are created using two CW dye lasers. The first laser is tuned to $589 \mathrm{~nm}$ to excite the $\mathrm{Na}(3 \mathrm{~s})$ to $\mathrm{Na}(3 \mathrm{p}) \mathrm{D} 2$ transition of sodium and the second laser is tuned to $569 \mathrm{~nm}$ to excite the formed $\mathrm{Na}(3 \mathrm{p})$ to the $\mathrm{Na}(4 \mathrm{~d})$ state. Their results showed the interesting importance of this process where they suggested that the collision cross section for this process is of the order of the cross-section for the atomic associative ionization collision of $\mathrm{Na}(4 \mathrm{~d})$ atoms with $\mathrm{Na}(3 \mathrm{~s})$ atoms.

This associative ionization reaction mechanism presents several possible applications. One of these applications is the study of cluster formation in atomic beams, where the formed $\mathrm{Na}_{3}^{+}$, may react with another $\mathrm{Na}$ atom (ground state or excited) to form $\mathrm{Na}_{4}^{+}$, etc. Besides, it can result in enhancing the population of seed electrons and in turn accelerates the plasma generation in sodium vapor. Meanwhile, it acts to deplete the saturated level $3 p$. Therefore, one expects that this reaction competes with both Penning and associative ionization processes.

Therefore, for further investigation of plasma generation in sodium vapor based on LIBORS technique, the present work is devoted to clarifying the role played by tri-atomic associative ionization as a seed electron generation process in plasma formation incomparable with those generated by associative, Penning, and photo-ionization mechanisms. The analysis is performed by adopting a model grounded on LIBORS technique. This model was previously developed [28] to interpret the experimental observation which is executed to study the associative ionization, and Penning ionization processes (atom-atom collisions). These are considered as main sources for seed electrons development in the ionization of sodium vapor irradiated by a CW laser source tuned to the transition 3s-3p prearranged by Caree el al. [29]. Then, this model is modified [30] and could successfully investigate the finding of the experimental observation given in [1] that carried out to measure to the first time the formation of tri-atomic ions in laser ionization of sodium vapor. 
As an extension to this work, the present study examines the ionization of sodium vapor irradiated by $\mathrm{CW}$ laser radiation taken into account the occurrence of various seed electron generation processes. The ionization mechanism proceeds as follows: 1) heating of the generated seed electrons through superelastic collisions, 2) initiation of electron impact ionization by the heated electrons leading to the development of new electrons. These electrons are then combined with steps (1) and (2) lead to ionization burnout which results in an almost complete ionization. These processes are compensated by loss of electrons from the interaction region through radiative and three body recombination processes. Thus, in this work, the computations paid great attention to study the individual consequence of each seed electron generation process to plasma formation. This study is achieved by analyzing the following parameters; electron energy distribution function (EEDF), the time evolution of; the electron density $\left(\mathrm{N}_{\mathrm{e}}\right)$, atomic ion density $\left(\mathrm{Na}^{+}\right)$, molecular ion density $\left(\mathrm{Na}_{2}^{+}\right)$, and trimer ions density $\left(\mathrm{Na}_{3}^{+}\right)$.

\section{Theoretical Backgrounds}

\subsection{Basic Formalism}

A detailed study of the theoretical background and modeling of ionization based on resonance saturation using tuned CW laser source is presented in our previous paper [28]. Sodium vapor has ionization energy, $E_{\text {ion }}$, greater than twice $\mathrm{E}_{12}$, which is the energy between the ground state $(3 \mathrm{~s})$ and the first excited state (the saturation level, $3 \mathrm{p}$ ), and less than the three times that energy, i.e., $E_{\text {ion }}<3.0$ $\mathrm{x} E_{12}$. This is a property of the alkali metal elements. Here, we recall that the modified LIBORS model [30] is applied to analyze plasma formation induced by pulsed laser radiation. The sodium atom is presumed to comprising 22 levels namely; a ground state, 18 excited states and three ionic states (atomic, molecular, and tri-atomic). Taken these assumption into consideration the following subsequent chain of ionization events take place in the interaction region: 1) two-photon ionization of the $3 p$ state, 2) single photoionization of the highly excited $\mathrm{nl}$ states 3) associative ionization of the $3 \mathrm{p}-3 \mathrm{p}$ states, 4) associative ionization of the $3 \mathrm{p}-3 \mathrm{~d}$ states, 5) Molnar-Hornbeck ionization in a collision between ground state and any of the highly excited states, 6) Penning ionization in a collision between the $3 p$ state and any of the highly excited states, 7) laser-induced Penning ionization a collision between two $3 p$ states in the presence of the laser photon, 8) electron impact excitation of electrons having energy $\varepsilon>E_{x}$ ( $E_{X}$ is the excitation energy from the ground or any of the excited states), 9) super-elastic collisions between highly excited states and low energy electrons $\varepsilon_{0}<\mathcal{E}$, 10) Collisional ionization by electrons of energy $\mathcal{E}>E_{\text {ion }}$, (where $\varepsilon$ is the total energy of the electron and is assumed to equal $\varepsilon=\left(\varepsilon_{1}\right)+\left(\varepsilon_{2}\right)+E_{\text {ion }}$ where $\varepsilon_{1}$ and $\varepsilon_{2}$ are the energies of the impinging and ejected electrons respectively), 11) energy pooling collisions between two $3 p$ states, 12) radiative recombination, 13) three-body recombination. To simplify the calculations, we 
have assumed that in the electron impact ionization and its inverse processes three-body recombination, the impinging and ejected electrons are assumed to carry the same amount of energy (i.e.) $\varepsilon_{1}=\varepsilon_{2}$.

\subsection{Rate Equations}

The above mentioned physical processes are encountered in the applied model given in Ref. 30 This model solves iteratively a set of rate equations that represent the time variation of the instantaneous population density of the various species present in the formed plasma. Besides, the model solves the Boltzmann equation for the electron energy distribution for the given normalization conditions. These are written as follows:

$$
\begin{aligned}
\frac{\mathrm{d} N(3 S)}{\mathrm{d} t}= & N(3 P)\left(R_{21}+A_{21}\right)-N(3 S) R_{12}+\int n_{e}(\varepsilon) N(3 P) K_{21}(\varepsilon) \mathrm{d} \varepsilon \\
& -\int n_{e}(\varepsilon) N(3 S) K_{12}(\varepsilon)+N(3 P) N(n) K_{P I}+\frac{1}{2} N^{2}(3 P) K_{E P} \\
& -N(n) N(3 S) K_{H M I}-N(3 S) n_{e}(\varepsilon) K_{1 c}(\varepsilon) \\
\frac{\mathrm{d} N(3 P)}{\mathrm{d} t}= & N(3 S) R_{12}-N(3 P)\left(R_{21}+A_{21}\right)-\int n_{e}(\varepsilon) N(3 P) K_{21}(\varepsilon) \mathrm{d} \varepsilon \\
+ & \int n_{e}(\varepsilon) N(3 S) K_{12}(\varepsilon) \mathrm{d} \varepsilon-\frac{1}{2} N^{2}(3 P) K_{\mathrm{AI} 1}-N(3 P) N(3 D) K_{\mathrm{AI} 2} \\
& -N(3 P) N(n) K_{\mathrm{PI}}-\frac{1}{2} N(3 P)^{2} \sigma_{\mathrm{PL}} v F-\frac{1}{2} N^{2}(3 P) K_{\mathrm{EP}} \\
& -N(3 P) \sigma_{2 c}^{(2)} F^{2}-N(3 S) n_{e}(\varepsilon) K_{2 c}(\varepsilon)-N(3 P) R_{26} \\
\frac{\mathrm{d} N(n)}{\mathrm{d} t}= & \sum_{n>m} n_{e}(\varepsilon) N(n) K_{n m}(\varepsilon)-\sum_{m \succ n} n_{e} N(n) K_{m n}(\varepsilon)-\sum_{n \succ m} A_{n m} N(n) \\
& -\sum_{n} n_{e}(\varepsilon) N(n) K_{n c}(\varepsilon)-\sum_{n} N(3 P) N(n) K_{\mathrm{PI}}+\frac{1}{2} N^{2}(3 P) K_{\mathrm{EP}} \\
& -\sum_{n} N(n) N(3 S) K_{\mathrm{HMI}}-\sum_{n \supset 2} N(n) \sigma_{n c}^{(1)} F \\
& +N_{\mathrm{Na}} n_{e}(\varepsilon) \sum_{n}\left[n_{e}(\varepsilon) K_{c n}(\varepsilon)+K_{R D}(\varepsilon)\right] \\
& +N(3 P) R_{26}-N(4 d) N\left(\mathrm{Na}_{2}\right) K_{4 d+\mathrm{Na} 2}
\end{aligned}
$$

The rate of growth of the molecular, atomic, and the tri-atomic ions is given by:

$$
\begin{gathered}
\frac{\mathrm{d} N\left(\mathrm{Na}_{2}^{+}\right)}{\mathrm{d} t}=\frac{1}{2} N^{2}(3 P) K_{\mathrm{AI}}+N(3 P) N(3 D) K_{\mathrm{AI} 2}+N(n) N(3 S) K_{\mathrm{HMI}} \\
\frac{\mathrm{d} N\left(\mathrm{Na}^{+}\right)}{\mathrm{d} t}= \\
+N(3 P) N(n) K_{\mathrm{PI}}+\frac{1}{2} N(3 P)^{2} \sigma_{\mathrm{PL}} \nu F \\
+N(3 P) \sigma_{2 c}^{(2)} F^{2}+\sum_{n \supset 2} N(n) \sigma_{n c}^{(1)} F \\
\frac{\mathrm{d} N\left(\mathrm{Na}_{3}^{+}\right)}{\mathrm{d} t}=N(4 d) N\left(\mathrm{Na}_{2}\right) K_{4 d+\mathrm{Na}_{2}}
\end{gathered}
$$

The time evolution of the electron energy distribution function is expressed 
by Boltzmann equation including all the processes which involve electrons interactions and written as:

$$
\begin{aligned}
\frac{\mathrm{d} n_{e}(\varepsilon)}{\mathrm{d} t}= & \sum_{m \supset n} n_{e}(\varepsilon) N(m) K_{n m}(\varepsilon)-\sum_{m \subset n} n_{e}(\varepsilon) K_{m n}(\varepsilon)+\sum_{n} n_{e}(\varepsilon) N(n) K_{n c}(\varepsilon) \\
& +\sum_{n} N(3 P) N(n) K_{\mathrm{PI}}+N(3 P) \sigma_{2 c}^{(2)} F^{2}+\sum_{n \supset 2} N(n) \sigma_{n c}^{(1)} F+\frac{1}{2} N^{2}(3 P) K_{\mathrm{AI} 1} \\
& +N(3 P) N(3 D) K_{\mathrm{AI} 2}+\frac{1}{2} N(3 P) v F+\sum_{n} N(n) N(3 S) K_{\mathrm{HMI}} \\
& -N_{\mathrm{Na}^{+}} n_{e}(\varepsilon) \sum_{n}\left[n_{e}(\varepsilon) K_{c n}(\varepsilon)+K_{\mathrm{RD}}(\varepsilon)\right]+N(4 d) N\left(\mathrm{Na}_{2}\right) K_{4 d+\mathrm{Na}_{2}}
\end{aligned}
$$

The normalization conditions considered in this analysis are taken as:

$$
\begin{gathered}
N_{0}=\sum_{n} N(n)+N\left(\mathrm{Na}^{+}\right) \\
\int_{0}^{\infty} n_{e}(\varepsilon) \varepsilon^{1 / 2} \mathrm{~d} \varepsilon=1, \quad \int_{0}^{\infty} n_{e}(\varepsilon) \mathrm{d} \varepsilon=N_{e}
\end{gathered}
$$

$N(3 s), N(3 p)$ and $N(n)$ represent the population density of the ground, first excited (saturated), and highly excited states respectively. $n_{e}(\varepsilon)$ refers to the free electron density of energy $\varepsilon$. $N_{0}$ is the density of $\mathrm{Na}$ vapor. F represents the photon flux density. $v$ is average velocity of atoms, in $\mathrm{cm} / \mathrm{sec}$.

Note that the factor $1 / 2$ appeared in the terms express the energy pooling and associative ionization mechanisms are added to correct for possible double counting of each colliding pair of identical particles [31].

The definition and source of the rate coefficients and cross sections of the collisional and photoionization processes appeared in the rate Equations (2) to (8) are defined in Table 1.

\section{Results and Discussions}

Equations (2) to (10) are incorporated into a computer program together with the relations which describe the rate coefficients and cross-sections of the physical processes encountered into the model and solved numerically applying Runge-Kutta fourth order technique. The calculations are performed under the experimental conditions given by Taplain and Smith [1]. In their experiment, the generation of sodium plasma induced by tuning a $\mathrm{CW}$ broadband dye laser to $589 \mathrm{~nm}$ to excite the Na3s $\left({ }^{2} \mathrm{~S}_{1 / 2}\right)$ to Na3p $\left({ }^{2} \mathrm{P}_{3 / 2}\right)$ transition and a CW frequency-stabilized narrow-band dye laser is tuned to $569 \mathrm{~nm}$ to excite the Na3p $\left({ }^{2} \mathrm{P}_{3 / 2}\right)$ to $\mathrm{Na} 4 \mathrm{~d}\left({ }^{2} \mathrm{D}_{5 / 2}\right)$. The applied laser beams of intensities up to $100 \mathrm{~W} / \mathrm{cm}^{2}$ are collimated and directed to the $\mathrm{Na}$ vapor chamber. The analysis considered atomic vapor density as $4.0 \times 10^{12} \mathrm{~cm}^{-3}$ at temperature $500-600 \mathrm{~K}$ for exposure time vary from 100 up to $500 \mathrm{~ns}$. The irradiated medium is found to evolve free electrons as well as atomic, molecular and trimer ions. Consequently, the study is devoted to examining the individual contribution of each process responsible for generating the seed electron and hence positive ions namely: associative

ionization, Penning ionization, photo-ionization, and tri-atomic associative 
Table 1. Definition of the rate coefficients and cross sections appeared in the rate equations.

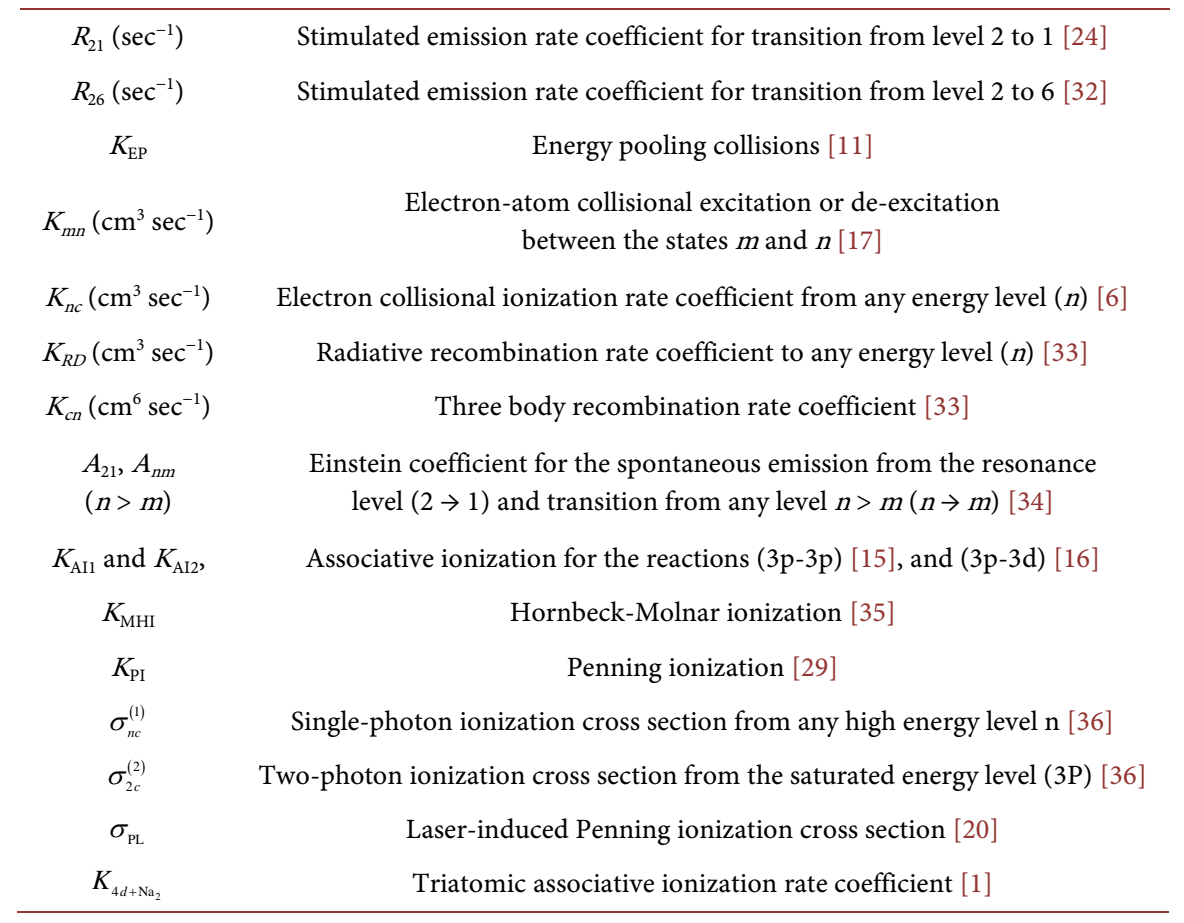

ionization to plasma formation. This investigation is obtained via a comparative study of the EEDF as well as the temporal evolution of electrons and ions density during the exposure time in the presence and absence of each of these ionization processes.

\subsection{Associative Ionization}

To examine the exact contribution of the associative ionization (AI) to the growth rate of the seed electrons calculations are carried out to obtain the EEDF in the presence and absence of this process for sodium atomic density $4 \times 10^{15}$ $\mathrm{cm}^{-3}$ and exposure time 500 ns. This is displayed in Figure 1 as curves (1) and (2) respectively. This figure demonstrates that the structure of the electron spectra has a non-Maxwellian distribution. Moreover, the effect of introducing the AI process (curve 1) appeared only in the low energy region $(0.0-0.6 \mathrm{eV})$ where an increase is observed in the peak labeled (a) at an electron energy of about 0.44 $\mathrm{eV}$. This increase reflects the role played by the AI process (produced by a collision of two Na3p atoms) in enriching the density of the low energy electrons. Beyond this energy range ( $0.6 \mathrm{eV}$ up to $5.25 \mathrm{eV})$ however, the structure of the electron spectra represented by both curves (1) and (2) coincides.

To clarify this result the temporal variation of the density of electrons corresponding to the low energy region $(0.0<\varepsilon<0.6 \mathrm{eV})$ in the presence (curve 1) and absence (curve 2) of AI is calculated and shown in Figure 2(a). It is noticed here that curve (1) lies much above the curve (2) over the whole exposure time duration. Also, the electron density grows at a constant rate. The obtained growth 


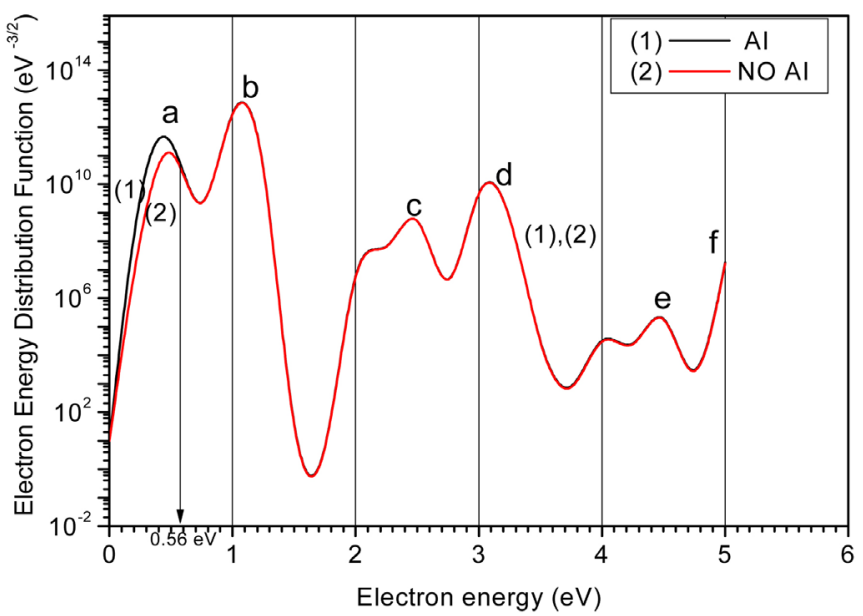

Figure 1. Comparison between the EEDF calculated in the presence (curve 1) and absence (curve 2) of associative ionization for the $\mathrm{Na}$ density $\mathrm{N}_{0}=4 \times 10^{15} \mathrm{~cm}^{-3}$ and exposure time $500 \mathrm{~ns}$.

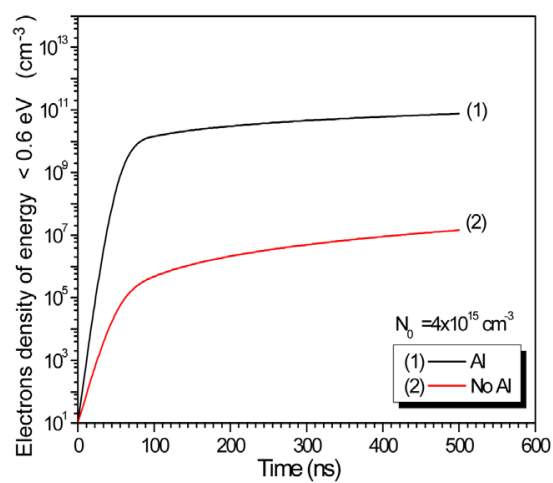

(a)

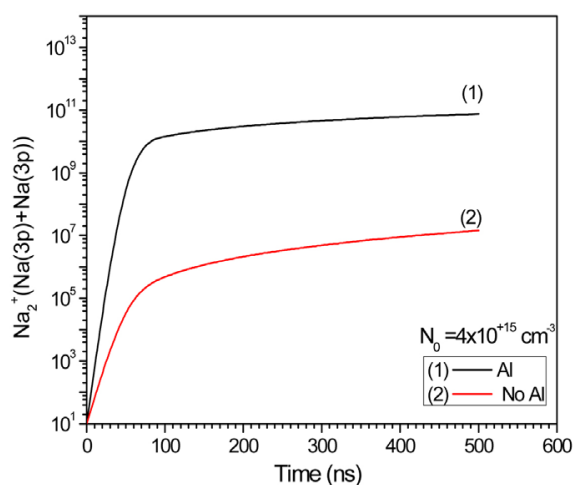

(b)

Figure 2. Temporal evolution of (a) density of electrons generated in the energy range $0.0<\varepsilon<0.6 \mathrm{eV}$, and (b) molecular ions density for $\mathrm{Na}$ atomic density $4.0 \times 10^{+15} \mathrm{~cm}^{-3}$ in the presence (curve 1) and absence (curve 2) of AI.

rate is found to be $\sim 5.17 \times 10^{3}$ at $t=500 \mathrm{~ns}$, which corresponds to an increase of the low energy electron density from $\sim 1.4 \times 10^{7} \mathrm{~cm}^{-3}$ to $\sim 8.0 \times 10^{10} \mathrm{~cm}^{-3}$. Figure 2(b) illustrates similar result but for the time variation of the formed molecular ions through AI process. Here curve (1) represents the presence of AI, and curve (2) is calculated in its absence. The similarity is shown in Figure 2(a) and Figure 2(b) for the time evolution of; electrons, and molecular ions respectively assured that $\mathrm{AI}$ is mainly responsible for the generation of electrons in the low energy range $(0.0-0.6 \mathrm{eV})$. Moreover, the high growth rate of these low energy electrons represented by curve (1) elucidated the significant role played by AI process in accelerating plasma generation.

\subsection{Penning Ionization and Photoionization}

In studying the effect of Penning ionization (PI) on the production of seed electrons, calculations showed that this process interplays with the photo-ionization 
process in line with the applied experimental conditions. This result could be attributed to the fact that the occurrence of both processes depends on the population of atoms in the highly excited states $(5 \mathrm{~s}, 4 \mathrm{~d} / 4 \mathrm{f}, 6 \mathrm{~s}$, and $5 \mathrm{~d})$. These states are mostly populated through energy pooling collisions of two $\mathrm{Na}(3 \mathrm{p})$ atoms. While the $4 \mathrm{p}$ and $3 \mathrm{~d}$ states are rather populated by radiative decay from these upper states which are produced by collisions [21] [37]. So, to account for the actual contribution of the PI process, a study is performed to compare the structure of the EEDF in the absence and presence of photo-ionization. This is displayed in Figure 3(a), and Figure 3(b) respectively. In this figure, the peaks appeared in the structure of the electron energy spectrum reflect the contribution of a specified physical process as shown in Table 2.

Figure 3(a) (absence of photoionization) shows that the structure of the calculated EEDF under the effect of PI (curve 1) is summarized as follows: decrease at the minimum position corresponds to electron energies $1.7 \mathrm{eV}$ and $3.6 \mathrm{eV}$ (PI and the first SEC of electrons generated by PI process respectively). An increase is shown at the peak (a) at energy $\sim 0.28 \mathrm{eV}$ (AI) followed by an increase in electron energies $0.5 \mathrm{eV}, 2.4 \mathrm{eV}$, and $4.4 \mathrm{eV}$. This performance of the EEDF can be explained based on the fact that electrons generated by AI $(0.28$ $\mathrm{eV}$ ) are heated up through the first $(2.4 \mathrm{eV})$ and second $(4.45 \mathrm{eV})$ SECs. Hence, they can undergo inelastic collisions and lose their energies in either exciting

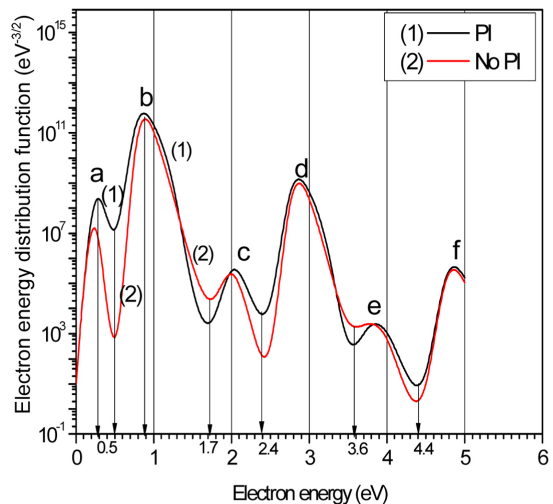

(a)

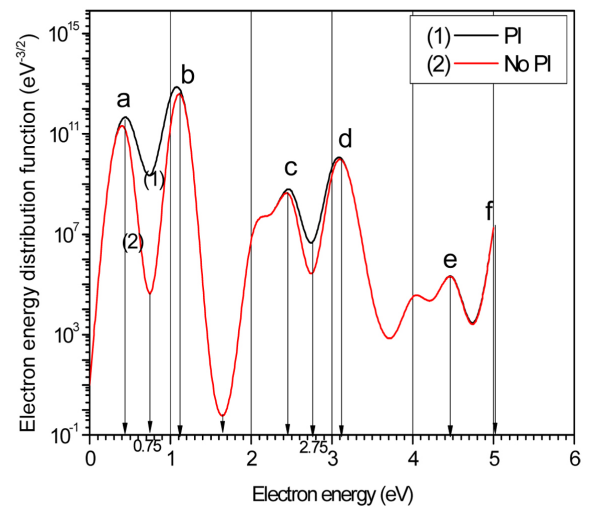

(b)

Figure 3. Effect of Penning ionization on the electron energy distribution function calculated in; (a) absence and (b) presence of photo-ionization process. Curves (1) and (2) represent the presence and absence of Penning ionization respectively.

Table 2. Physical mechanisms, and the corresponding Peak label.

\begin{tabular}{cc}
\hline Peak labeled & Physical mechanism \\
\hline$a$ & Associative ionization (AI) \\
$c$ & Penning Ionization (PI) \\
$d$ & $1^{\text {st }}$ Super elastic collision (electrons generated by AI) \\
$e$ & $1^{\text {st }}$ Super elastic collision (electrons generated by PI) \\
$f$ & $2^{\text {nd }}$ Super elastic collision (electrons generated via AI) \\
\hline
\end{tabular}


ground state atoms or ionize the populated excited states. These processes lead to the generation of low energy electrons. This, in turn, could elucidate the increase of the EEDF corresponds to the peak (a) in the presence of PI process.

On the other hand, to clarify the role played by the PHI process on the plasma development Figure 3(b) illustrates the calculated EEDF in the presence of this process under the action and omission of the PI process. This figure showed an increase of the values of the peaks (a to f) with a shift of the whole electron energy spectrum towards the high energy regime in the presence and absence of PI as displayed by curves (1), and (2) respectively. This result is depicted in Table 3. Moreover, It is shown from this figure that, despite the growth of the values of the minima appeared at energies $\sim 0.75 \mathrm{eV}$ and $\sim 2.75 \mathrm{eV}$, there is a clear similarity in the structure of the EEDF represented by the curves (1 and 2). This result is investigated following to the fact that, PI acts only to enhance the generation of the electrons density carrying energy in the range $0.75 \mathrm{eV}<\varepsilon<$ $1.25 \mathrm{eV}$. These electrons are subsequently heated up through super-elastic collision (SEC) with $\mathrm{Na}(3 \mathrm{p})$ atoms. Those excited atoms boost the kinetic energy of the created electrons by $2.1 \mathrm{eV}$. This, in turn, enhances the population of electrons having the energy of about $\sim 2.75 \mathrm{e} \mathrm{V}$. Over the remaining energy range $(2.75 \mathrm{eV}<\varepsilon<5.0 \mathrm{eV})$ both curves (1, and 2) are almost found to coincide. The behavior displayed in this figure is demonstrated in Table 3 which undertaking the pronounced effect of the PHI process on the rate of electron production and its interplay with the PI process. Bearing in mind that, the occurrence of both mechanisms mainly depends on the population of the same highly excited states. This result is assured from the fast growth of the EEDF obtained at the first peak (a) under the effect of PI and absence of PHI given in Table 3. Consequently, in dealing with PI process, one should take into account the competing role between PI and photo-ionization processes in relation to the experimental condition under investigation.

As we have seen above, the mutual effect of photo-ionization and PI processes leads to a change in the electron energy structure. Accordingly, it was necessary to have a detailed study on the real impact of the photoionization process on the

Table 3. Peak label, peak energy position, EEDF calculated under the effect and ignorance of PI for the absence (a) and presence (b) of photoionization process.

\begin{tabular}{ccccccc}
\hline & \multicolumn{3}{c}{ Absence of PHI (a) } & \multicolumn{3}{c}{ Presence of PHI (b) } \\
\cline { 2 - 7 } Peak labeled & $\begin{array}{c}\text { Electron } \\
\text { Energy } \\
(\mathrm{eV})\end{array}$ & $\begin{array}{c}\text { EEDF in the } \\
\text { presence PI }\end{array}$ & $\begin{array}{c}\text { EEDF in the } \\
\text { absence PI }\end{array}$ & $\begin{array}{c}\text { Electron } \\
\text { Energy } \\
(\mathrm{eV})\end{array}$ & $\begin{array}{c}\text { EEDF in the } \\
\text { presence PI }\end{array}$ & $\begin{array}{c}\text { EEDF in the } \\
\text { absence PI }\end{array}$ \\
\hline a & $0.28 \mathrm{eV}$ & $2.7 \times 0^{8}$ & $1.77 \times 10^{7}$ & $0.44 \mathrm{eV}$ & $5.68 \times 10^{11}$ & $1.9 \times 10^{11}$ \\
$\mathrm{~b}$ & $0.88 \mathrm{eV}$ & $8.55 \times 0^{11}$ & $3.29 \times 10^{11}$ & $1.14 \mathrm{eV}$ & $1.3 \times 10^{13}$ & $3.8 \times 10^{12}$ \\
$\mathrm{c}$ & $2.06 \mathrm{eV}$ & $5.82 \times 10^{5}$ & $2.24 \times 10^{5}$ & $2.48 \mathrm{eV}$ & $1.07 \times 10^{9}$ & $2.7 \times 10^{8}$ \\
$\mathrm{~d}$ & $2.9 \mathrm{eV}$ & $2.43 \times 10^{9}$ & $1.08 \times 10^{9}$ & $3.12 \mathrm{eV}$ & $2.8 \times 10^{10}$ & $2.8 \times 10^{10}$ \\
$\mathrm{e}$ & $3.86 \mathrm{eV}$ & $3.27 \times 10^{3}$ & $2.49 \times 10^{3}$ & $4.45 \mathrm{eV}$ & $2.2 \times 10^{5}$ & $2.2 \times 10^{5}$ \\
$\mathrm{f}$ & $4.87 \mathrm{eV}$ & $4.77 \times 10^{5}$ & $4.77 \times 10^{5}$ & $5.0 \mathrm{eV}$ & $2.67 \times 10^{7}$ & $2.67 \times 10^{7}$ \\
\hline
\end{tabular}


temporal variation of both; generated free electrons, and corresponding atomic ions. Figure 4 and Figure 5 demonstrate the effect of photo-ionization on these two parameters respectively. The effect of presence (curve 1) and absence (curve 2) of photoionization mechanism on the electron growth rate during the exposure time are illustrated in Figure 4. The examined exposure time is extended from 100 ns to 500 ns. From this study, it is observed that PHI process plays some degree of enriching the electron density. Where the electron density is increased from $\sim 3.0 \times 10^{12} \mathrm{~cm}^{-3}$ to $7.0 \times 10^{13} \mathrm{~cm}^{-3}$ at exposure time $t=500 \mathrm{~ns}$. This increase could be an important factor for fast plasma development. On the other hand, since each electron ejected from an atom leaves behind a positive atomic ion, therefore, Figure 5 presents the comparison between the time evolution of

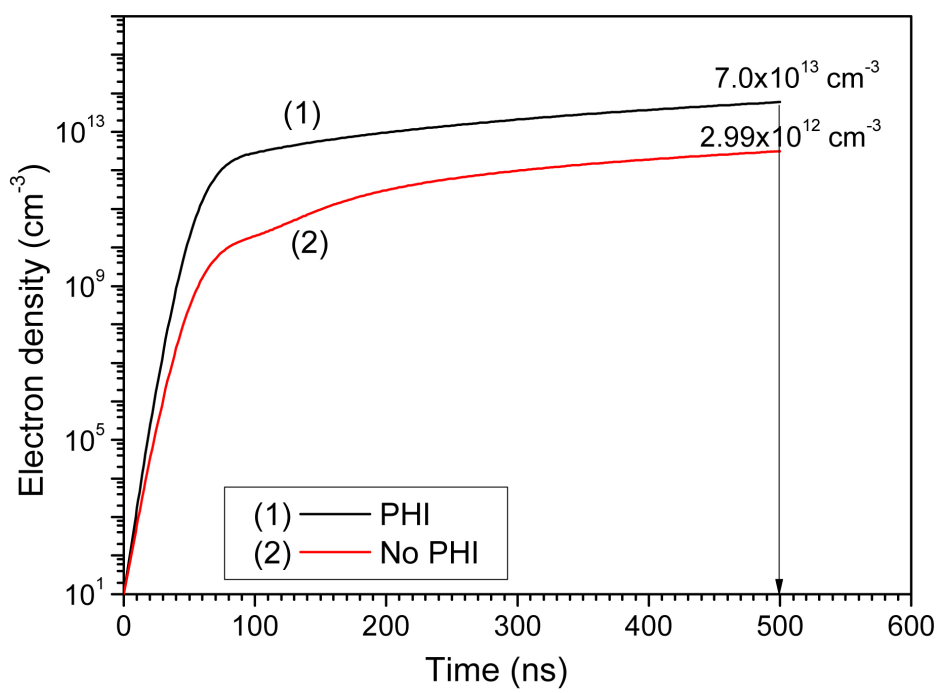

Figure 4. Time evolution of electron density in the presence (curve 1) and absence (curve 2 ) of the photoionization process.

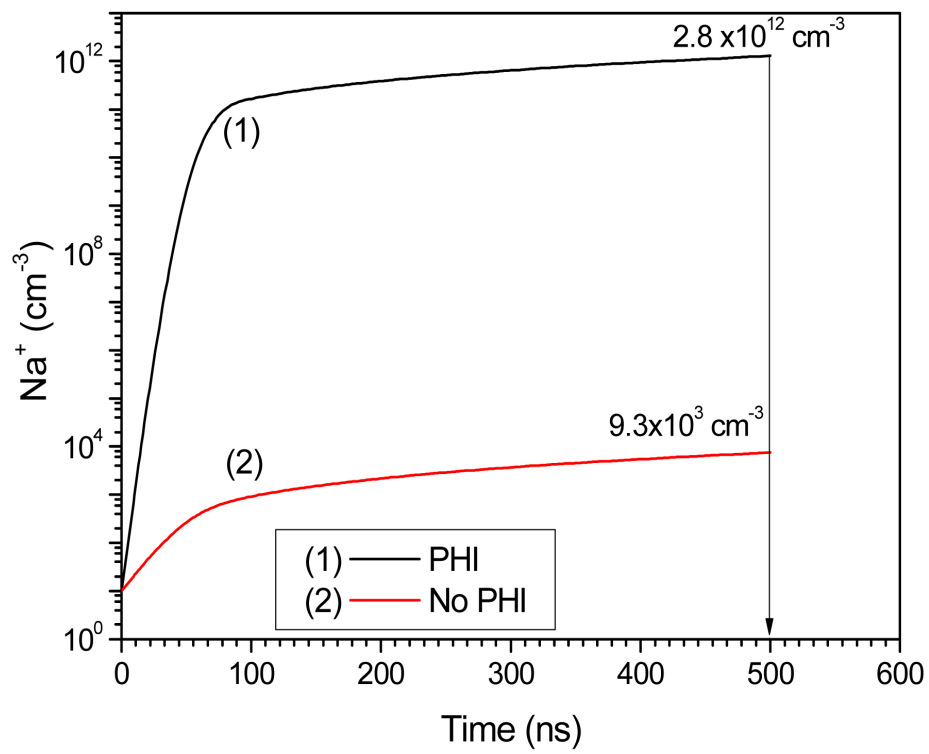

Figure 5. Same as in Figure 4 but for the atomic ions density. 
the formed atomic ions in the interaction region in the presence (curve 1) and absence (curve 2) of photoionization mechanism. The behavior shown in this figure evidences those atomic ions are produced through interactions other than photoionization in contrast to the molecular ions shown in Figure 2(b) which are produced mainly through AI process. A ratio of $\sim 10^{8}$ is obtained for the atomic ion density obtained in the presence (curve 1) and absence (curve 2) of different interactions for an exposure time $500 \mathrm{~ns}$. This result proves that the presence of photoionization enhances the ignition of different ionizing mechanisms which act to release a reasonable density of atomic ions. These mechanisms could be mainly devoted to the electron impact ionization of the highly excited state with the low energies electrons ejected by PHI.

\subsection{Tri-Atomic Associative Ionization}

According to what mentioned above, this process results in the interaction between atoms in the $4 \mathrm{~d}$ state and ground state molecules and leads to the formation of triatomic ions and free electrons. So, for this process to take place the $\mathrm{Na}(4 \mathrm{~d})$ state should be highly populated. This is the role played by the $569 \mathrm{~nm}$ laser source which is tuned to the transition $3 \mathrm{p}-4 \mathrm{~d}$ in the experimental measurement under investigation [1]. Figure 6 indicates a comparison between the calculated EEDF in the presence of tri-atomic associative ionization process (curve 1) and its absence (curve 2). It is noticed from this figure that regardless the decrease observed at the minimum positions $0.75 \mathrm{eV}$ and $1.7 \mathrm{eV}$, introducing this process enhances the peak values of the electron energy spectrum. This enhancement is attributed to the fact that the overpopulation of the $4 \mathrm{~d}$ state possibly activates the occurrence of some ionization processes that are governed by this state. The outcome of such processes is laying in either the enhancement of

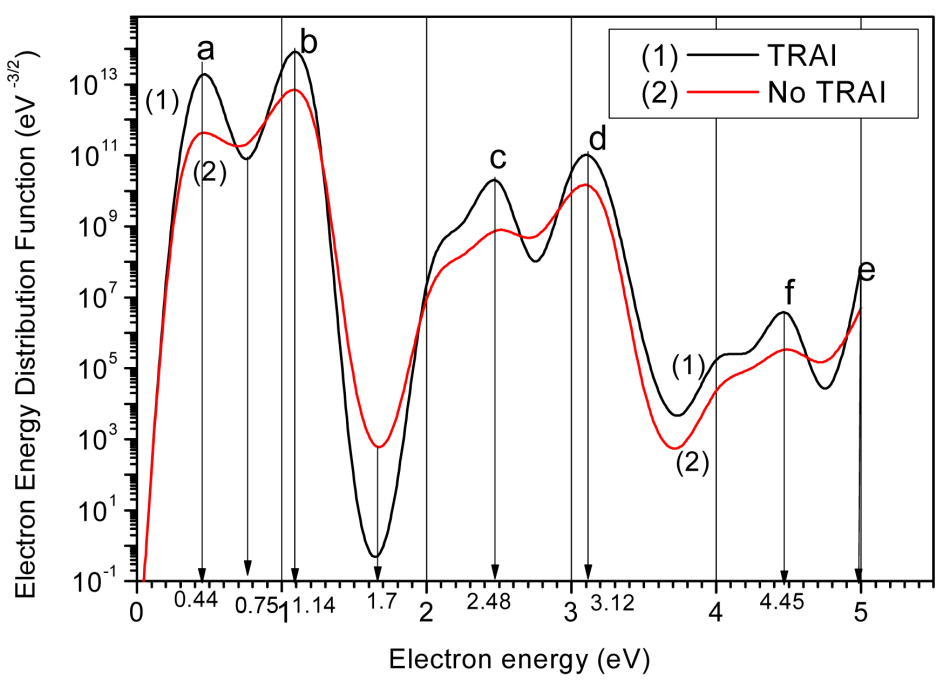

Figure 6. Electron energy distribution function calculated for atomic sodium density of $4 \times 10^{15} \mathrm{~cm}^{-3}$ and exposure time $500 \mathrm{~ns}$ in the presence (curve 1) and absence (curve 2) of tri-atomic associative ionization process. 
the highly excited state's density (energy pooling) followed by their photoionization, or the generation of newly born electrons (Penning ionization, Hornbeck-Molnar, and photoionization). On the other hand, the lowering of the EEDF appeared at $0.75 \mathrm{eV}$, and $1.75 \mathrm{eV}$ (the energy range corresponds to PI process) could be attributed to the fact that the population of the $4 \mathrm{~d}$ state depends mainly on the population of the $3 p$ state. The latter state is the foremost source required for the occurrence of a PI process.

Since those mentioned ionization processes are resulting in free electrons generation and hence atomic ions formation, therefore the effect of tri-atomic associative ionization process on the time evolution of 1) the electron density, and 2) the atomic ions density is examined and shown in Figure 7. In this figure curve (1) refers to the presence of this process, while curve (2) represents its

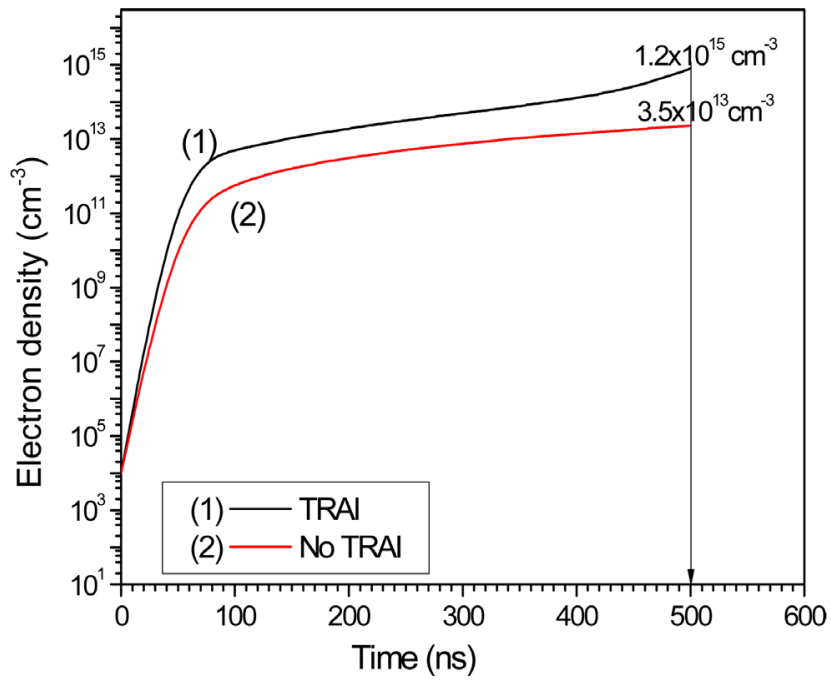

(a)

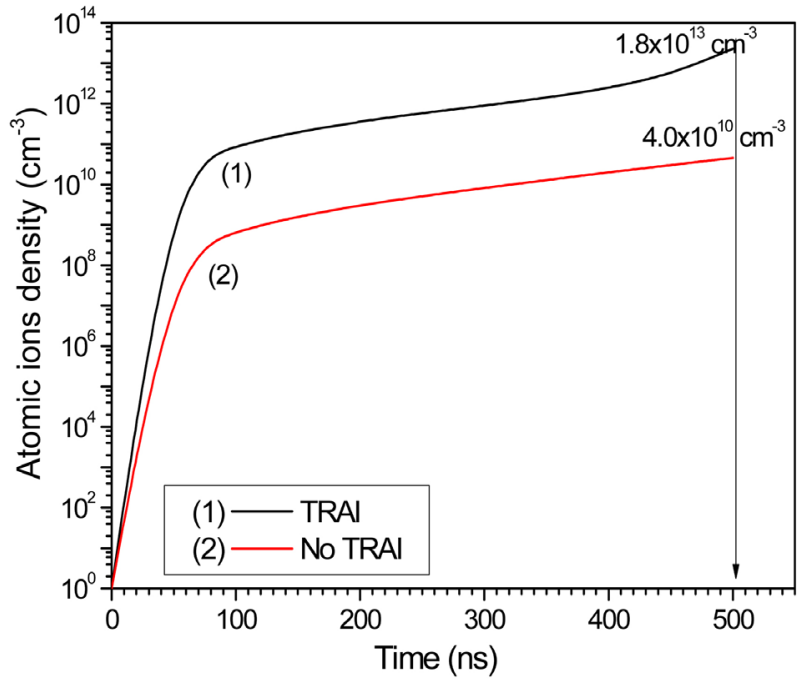

(b)

Figure 7. Effect of the presence (curve 1) and absence (curve 2) of tri-atomic associative ionization on the time evolution of (a) electron density (b) atomic ions density. 
absence. It is clear from Figure 7(a) that the growth rate of the electrons density shown by curve (1) lies above those represented by curve (2) with a slightly faster increasing rate is observed at exposure time $500 \mathrm{~ns}$. The increasing rate is found to change from $\sim 14$ to $\sim 34$. Furthermore, a corresponding increase in the atomic ions density is shown in Figure 7 (b) in the presence of tri-atomic associative ionization (curve 1) at exposure time 500 which is estimated to be about 468 times those represented by curve (2). This increase is attributed to the high ionization rate of the $4 \mathrm{~d}$ state due to the contribution of the ionization processes sharing the $4 \mathrm{~d}$ state.

\section{Conclusions}

In the present work, using a modified model based on LIBORS technique, the results of calculations of the EEDF in the presence and absence of each of the seed electron generation processes namely: Associative ionization, Penning ionization, photoionization, and Tri-atomic associative ionization showed the non-Maxwellian distribution of the electrons. Besides, the growth rate of electrons which reflects the ions formation is achieved by comparing the electron (ions) density in the presence and absence of each of the considered ionization processes. This leads to the following conclusion:

- Associative ionization results only in an increase of the electron density by an order of $\approx 10^{4}$ on the energy range $0.0-0.25 \mathrm{eV}$. This, in turn, represents a fair contribution to plasma development. The similar growth rate is obtained for the corresponding molecular ions density.

- Penning ionization and photoionization are found to signify two strongly competing processes both sharing the same highly excited states which are populated via energy pooling process. The later process diminishes the contribution of the Penning ionization to the plasma generation. Consequently, in studying the role of Penning ionization process, photoionization process is ignored.

- Photoionization showed a pronounced effect on the enhancement of the atomic ions density during the examined exposure time range. Besides, the peaks of the EEDF showed an observable increase, and their position undergoes slight shifting towards the high-energy region. This shift points out the increase in the density of the high-energy electrons.

- The formation of Tri-atomic associative ionization is found to play a vital role in enhancing the seed electron population which in turn accelerates the plasma generation (less exposure time). Besides, it increases the possibility of studying cluster formation in atomic beams, for example, the formed $\mathrm{Na}_{3}^{+}$, may react with another $\mathrm{Na}$ atom (ground state or excited) to form $\mathrm{Na}_{4}^{+}$. These clusters may found several applications.

\section{References}

[1] Tapalian, C. and Smith, W.W. (1993) Chemical Physics Letters, 211, 425-429. 
https://doi.org/10.1016/0009-2614(93)87085-H

[2] Lucatorto, T.B. and McIlrath, T.B. (1976) Physical Review Letters, 37, 428-431. https://doi.org/10.1103/PhysRevLett.37.428

[3] Barbier, C.M., Lindinger, L. and Deloche, R. (1982) Journal of Physics B: Atomic and Molecular Physics, 15, 3463.

[4] Vesa, D. and Sansonetti, Z. (1992) Physik D Atoms, Molecules and Clusters, 22, 463.

[5] Skenderovic, H., Labazan, I., Milosevic, S. and Pichler, G. (2000) Physical Review A, 62, Article ID: 052707.

[6] Kallenbach, A. and Kock, M. (1989) Journal of Physics B, 22, 1691.

[7] Bearman, G.H. and Leventhal, J.J. (1978) Physical Review Letters, 41, 1227-1230. https://doi.org/10.1103/PhysRevLett.41.1227

[8] Jong, A.D. and Valk, F.V.D. (1979) Journal of Physics B: Atomic and Molecular Physics, 12, L561. https://doi.org/10.1088/0022-3700/12/18/006

[9] Kushawaha, V.S. and Leventhal, J.J. (1980) Physical Review A, 22, 2468-2473. https://doi.org/10.1103/PhysRevA.22.2468

[10] Roussel, F., Breger, P., Spiess, G., Manus, C. and Geltman, S. (1980) Journal of Physics B: Atomic and Molecular Physics, 13, L631.

[11] Carre, B., Roussel, F., Breger, P. and Spiess, G. (1981) Journal of Physics B: Atomic and Molecular Physics, 14, 4289-4300.

[12] Weiner, J. and Polak-Dingels, P. (1981) The Journal of Chemical Physics, 74, 508-511. https://doi.org/10.1063/1.440858

[13] Kushawaha, S.V. and Leventhal, J.J. (1982) Physical Review A, 25, 346-350. https://doi.org/10.1103/PhysRevA.25.346

[14] Babenko, E., Tapalian, C. and Smith, W.W. (1995) Chemical Physics Letters, 244, 121-126. https://doi.org/10.1016/0009-2614(95)00878-8

[15] Allegrini, M., Alzetta, G., Kopystynska, A., Moi, L. and Orriols, G. (1976) Optics Communications, 19, 96-99. https://doi.org/10.1016/0030-4018(76)90394-1

[16] Huennekens, J. and Gallagher, A. (1983) Physical Review A, 27, 771-784.

[17] Mahmoud, M.A. (2005) Journal of Physics B: Atomic, Molecular and Optical Physics, 38, 1545-1556. https://doi.org/10.1088/0953-4075/38/10/012

[18] Mahmoud, M.A. and Gamal, Y.E.E. (2012) Indian Journal of Physics, 86, 659-666. https://doi.org/10.1007/s12648-012-0093-3

[19] Carre, B., Roussel, F., Spiess, G., Bizau, J.M., Gerard, P. and Wuilleumier, F. (1986) Zeitschrift für Physik D Atoms, Molecules and Clusters, 1, 79. https://doi.org/10.1007/BF01384662

[20] Stacewicz, T., Gorbunov, N.A. and Kozlowski, P. (1998) Applied Physics B, 66, 461-465. https://doi.org/10.1007/s003400050419

[21] Measures, R.M. and Cardinal, P.G. (1981) Physical Review A, 23, 804-815.

[22] Measures, R.M., Drewell, N. and Cardinal, P. (1979) Journal of Applied Physics, 50, 2662-2669. https://doi.org/10.1063/1.326223

[23] Leonov, A.G., Chekhov, D.I. and Starostin, A.N. (1997) JETP, 84, 703-715. https://doi.org/10.1134/1.558204

[24] Gorbunov, N.A., Grochola, A., Kruk, P., Pietruczuk, A. and Stacewicz, T. (2002) Plasma Sources Science and Technology, 11, 492-497. https://doi.org/10.1088/0963-0252/11/4/316 
[25] Kosarev, N.I. and Shaparev, N.Ya. (2006) Quantum Electronics, 36, 369-375. https://doi.org/10.1070/QE2006v036n04ABEH013153

[26] Stacewicz, T. and Topulos, G. (1988) Physica Scripta, 38, 4, 560-563. https://doi.org/10.1088/0031-8949/38/4/010

[27] Stacewicz, T. and Latek, W. (1990) Physica Scripta, 42, 658-660. https://doi.org/10.1088/0031-8949/42/6/005

[28] Mahmoud, M.A. and Gamal, Y.E.E. (1995) Journal of the Physical Society of Japan, 64, 4653-4659. https://doi.org/10.1143/JPSJ.64.4653

[29] Carre, B., Spiess, G., Picque, J.M., Dhes, P., Gerared, P., Wuilleumeir, F., Keller, J.C., Le Gouet, J., Picque, J.L., Ederer, D.L. and Koch, P.M. (1984) Optics Communications, 52, 29. https://doi.org/10.1016/0030-4018(84)90068-3

[30] Abdelati, M.A., Mahmoud, M.A. and Gamal, Y.E.E. (2014) Journal of Applied Mathematics and Physics, 2, 1123-1129. https://doi.org/10.4236/jamp.2014.212131

[31] Bezuglov, N.N., Klyucharev, A.N. and Sheverev, V.A. (1987) Journal of Physics B: Atomic and Molecular Physics, 20, 2497-2513. https://doi.org/10.1088/0022-3700/20/11/018

[32] Drawin, H.N. and Felenbok, P. (1965) Data for Plasma in Local Thermodynamic Equilibrium. Gauthier-Villarrs, Paris.

[33] Vriens, J.L. and Smeets, A.H. (1980) Physical Review A, 22, 940. https://doi.org/10.1103/PhysRevA.22.940

[34] Measures, R., Wong, S.K. and Cardinal, P.G. (1982) Journal of Applied Physics, 53, 5541-5551. https://doi.org/10.1063/1.331489

[35] Babenko, E., Tapalian, C. and Smith, W.W. (1995) Chemical Physics Letters, 244, 121-126. https://doi.org/10.1016/0009-2614(95)00878-8

[36] Laughlin, C. (1978) Journal of Physics B: Atomic and Molecular Physics, 11, 1399-1412. https://doi.org/10.1088/0022-3700/11/8/011

[37] Horvatic, V. (2003) FIZIKA A, 12, 97-114. 